\title{
Diagnostic Accuracy of MRI in Primary Cervical Cancer
}

\author{
Giuliano Rigon ${ }^{1}$, Cristina Vallone ${ }^{1}$, Andrea Starita ${ }^{1}$, Marco Flavio Michele Vismara ${ }^{2}$, \\ Pasquale Ialongo $^{3}$, Lorenza Putignani ${ }^{4^{*}}$, Fabrizio Signore ${ }^{{ }^{*}}$ \\ ${ }^{1}$ Department of Obstetrics and Gynaecology, S. Camillo-Forlanini Hospital, Rome, Italy \\ ${ }^{2}$ Department of Molecular Medicine, Faculty of Medicine and Pharmacy, "Sapienza" University of Rome, Rome, Italy \\ ${ }^{3}$ Department of Radiology, S. Camillo-Forlanini Hospital, Rome, Italy \\ ${ }^{4}$ Microbiology Unit, Children's Hospital and Research Institute "Bambino Gesư", Rome, Italy \\ Email: ${ }^{*}$ ssignore@scamilloforlanini.rm.it, lorenza.putignani@opbg.net
}

Received January 14, 2012; revised February 12, 2012; accepted February 22, 2012

\begin{abstract}
Introduction: Magnetic resonance imaging (MRI) studies obtained during the initial staging of patients affected by uterine cervical cancer were compared to the final histological report after surgery. Methods: Data were retrieved from published papers. Results: MRI detection of lymph node metastases shows a sensitivity of 49.3\% (1209 patients) and a specificity of $87.7 \%$ (1182 patients). Parametrial involvement detection has $66.2 \%$ sensitivity (1288 patients) and $83.6 \%$ specificity (1282 patients). MRI tumor size evaluation shows significant error. Even detection of over $1 \mathrm{~cm}$ diameter primary tumor can fail. MRI appears promising in the detection of myometrial and endometrial involvement. Conclusions: Primary uterine cervical cancer evaluation with routine MRI has a limited accuracy especially in the detection of lymph node involvement and parametrial invasion. It is not sensitive enough to replace histology of dissected nodes and parametria. Tumor size estimation is imprecise. Detection of myometrial and endometrial invasion using MRI might be possible. Awareness of MRI limitations is crucial in primary cervical cancer staging.
\end{abstract}

Keywords: Cervical Cancer; MRI; Parametrial; Lymph Node Involvement

\section{Introduction}

Uterine cervical cancer is the third most common gynaecologic malignancy reported in the United States [1]. In 200711,150 new cases were diagnosed and 3670 deaths were expected in the United States [1]. Currently, the International Federation of Gynecology and Obstetrics (FIGO) staging for cervical carcinoma is primarily based on physical examination. Staging errors are strongly associated with poor prognosis [1]. Exclusive FIGO clinical staging has been reported as accurate in only $29 \%$ of cases [2].

FIGO staging does not include lymph node status and tends to underestimate in $20 \%-30 \%$ of low stage cervical cancer patients when compared to surgical staging. FIGO understaging has been reported in up to $23 \%$ of stage IIb disease, and $40 \%$ to $64 \%$ in stage IIIb. About $40 \%$ of the patients receive an inaccurate preoperative staging [3].

Together with parametrial extension and positive surgical margins, one of the most important prognostic factors in cervical cancer is the presence of lymph node metastasis.

Imaging technologies, as Computed Assisted Tomo-

"Corresponding author. graphy (CAT), Magnetic Resonance Imaging (MRI) and Positron Emission Tomography (PET) might have an impact in determining the proper treatment for patients with cervical cancer.

International papers screened using PubMed were reviewed. Sensitivity and specificity of routine MRI for 1) primary tumour detection; 2) internal of involvement; 3) myometrial invasion; 4) lymph node involvement; 5) parametrial invasion; 6) bladder invasion; 7) rectal invasion and 8) vaginal invasion were noted. MRI tumor size evaluation was compared to histologic tumor size. Radiological findings were compared to the postoperative histological report.

\section{Methods}

\subsection{Literature Search and Study Selection}

This study was approved by San Camillo-Forlanini Institutional Review Board.

A literature search of international studies was carried out to identify articles on MRI preoperative diagnostic performance in patients with cervical carcinoma. MEDLINE database (2000 to 2011) was searched for the following terms: "cervical cancer, cervical neoplasm, MRI, parametrial and lymph nodes" as medical subject head- 
ings (MeSH) and "specificity and sensitivity" as text words. Only original articles with raw data including comparison between immediately preoperative MRI results and post-operative histopathology were included. Eighty two papers were found to be eligible and 40 fulfilled inclusion criteria at least partially. Only 24 papers had complete data on lymphnode extension and/or parametrial invasion.

\subsection{Data Evaluation}

Two observers independently performed data evaluation, and discrepancies in judgement were solved by the independent appraisal of a third reviewer. The following items were extracted: 1) year of publication; 2) MRI technique (most authors used T1 weighted images [T1w] and $\mathrm{T} 2$ weighted images [T2w]; some of them compared different signals $[4,5]$, or did not specify MRI techniques [6,7]; best results were used for analysis); 3) total sample size; 4) presence or absence of tumor in sites like lymph nodes, parametria, etc.; 5) and tumor size data indicated in centimeters.
Sensitivity and specificity values for lymph node and parametrial invasion (Tables 1-2), expressed as percentage, rounded up/down to the first significant digit, were calculated from published data.

\section{Results}

Table 1 reports the sensitivity and specificity of routine MRI in detecting lymph node metastases in case of cervical cancer (1444 patients). Overall sensitivity is $51.4 \%$ and specificity is $86.6 \%$. Table 2 reports MRI sensitivity and specificity in detecting parametrial invasion in cervical cancer (1341 patients). Overall sensitivity is $66.2 \%$ and specificity is $83.8 \%$.

As to other endpoints, like detection of invasion of proximal structures, data were less homogeneous because of limited numbers or differences in study design.

Invasion of the serosal and muscle layer of the bladder. Rockall et al., highlighted 13 false positives out of 112 patients with an overall sensitivity of $100.0 \%$ and a specificity of $88.0 \%$ for bladder invasion detection with MRI [6]. Hertel et al., calculated a sensitivity of $64.0 \%$

Table 1. MRI detection of lymph node metastases: computation of sensitivity and specificity values.

\begin{tabular}{|c|c|c|c|c|}
\hline Patient $\left(\mathrm{N}^{\circ}\right)$ & Sensitivity (\%) & Patient $\left(\mathrm{N}^{\circ}\right)$ & Specificity (\%) & Reference \\
\hline 101 & 36.0 & 101 & 84.0 & {$[22]$} \\
\hline 150 & 37.0 & 150 & 92.0 & {$[15]$} \\
\hline 55 & 33.3 & 55 & 97.4 & {$[27]$} \\
\hline 119 & 89.0 & 119 & 67.0 & [20] \\
\hline 172 & 37.0 & 172 & 94.0 & {$[11]$} \\
\hline 36 & 57.0 & 36 & 73.0 & [21] \\
\hline 44 & 27.0 & 44 & 94.0 & [23] \\
\hline 113 & 36.0 & 113 & 97.0 & [12] \\
\hline 92 & 73.0 & 92 & 93.8 & [40] \\
\hline 109 & 25.0 & 109 & 87.0 & [7] \\
\hline 41 & 82.0 & 41 & 87.0 & [13] \\
\hline 27 & 50.0 & - & - & [28] \\
\hline 22 & 38.5 & 22 & 44.4 & {$[41]$} \\
\hline 62 & 72.9 & 62 & 93.1 & {$[31]$} \\
\hline 31 & 71.0 & 31 & 92.0 & [14] \\
\hline 35 & 73.0 & 35 & 83.0 & [29] \\
\hline 99 & 77.7 & 99 & 85.6 & [17] \\
\hline 53 & 33.0 & 53 & 92.0 & [24] \\
\hline 83 & 64.3 & 83 & 69.1 & {$[6]$} \\
\hline $1444^{\mathrm{a}}$ & $51.4^{\mathrm{b}}$ & $1417^{\mathrm{c}}$ & $86.6^{\mathrm{d}}$ & This work \\
\hline
\end{tabular}

${ }^{\mathrm{a}}$ The number indicates the total number of patients; ${ }^{\mathrm{b}}$ The number is obtained as $\Sigma$ (patient ${ }_{\mathrm{n}} \times$ sensitivity $\left._{\mathrm{n}}\right) / 1444$ and represents the overall sensitivity; ${ }^{\mathrm{c}}$ The number indicates the total number of patients; ${ }^{\mathrm{d}}$ The number is obtained as $\Sigma$ (patient $\mathrm{n}_{\mathrm{n}} \times$ sensitivity $_{\mathrm{n}}$ )/1417 and represents the overall specificity. 
Table 2. MRI detection of parametrial invasion: computation of sensitivity and specificity values.

\begin{tabular}{ccccc}
\hline Patient $\left(\mathbf{N}^{\circ}\right)$ & Sensitivity $\mathbf{( \% )}$ & Patient $\mathbf{( \mathbf { N } ^ { \circ } )}$ & Specificity $\mathbf{( \% )}$ & Reference \\
\hline 101 & 52.0 & 101 & 63.0 & {$[22]$} \\
- & - & 150 & 97.0 & {$[15]$} \\
119 & 80.0 & 119 & 91.3 & {$[20]$} \\
112 & 100.0 & - & - & {$[6]$} \\
172 & 53.0 & 172 & 75.0 & {$[11]$} \\
44 & 21.0 & - & - & {$[23]$} \\
113 & 38.0 & 113 & 99.0 & {$[12]$} \\
85 & 77.8 & 85 & 88.2 & {$[13]$} \\
41 & 97.0 & 41 & 91.0 & {$[4]$} \\
75 & 100.0 & 75 & 93.0 & {$[8]$} \\
103 & 60.0 & 103 & 73.0 & {$[33]$} \\
159 & 59.3 & 159 & 61.5 & {$[14]$} \\
31 & 100.0 & 31 & 96.0 & {$[5]$} \\
38 & 90.0 & 38 & 92.0 & {$[18]$} \\
95 & 50.0 & 95 & 97.8 & {$[24]$} \\
53 & 67.0 & 53 & 92.0 & This work \\
\hline $1341^{\mathrm{a}}$ & $66.2^{\mathrm{b}}$ & $1335^{\mathrm{a}}$ & $83.8^{\mathrm{b}}$ & \\
\hline
\end{tabular}

${ }^{\mathrm{a}}$ The number indicates the total number of patients; ${ }^{\mathrm{b}}$ The number is obtained as $\Sigma\left(\right.$ patient $_{\mathrm{n}} \times$ sensitivity $\left._{\mathrm{n}}\right) / 1341$ and represents the overall sensitivity; ${ }^{\mathrm{c}}$ The number indicates the total number of patients; ${ }^{\mathrm{d}}$ The number is obtained as $\Sigma\left(\right.$ patient $_{\mathrm{n}} \times$ sensitivity $\left._{\mathrm{n}}\right) / 1335$ and represents the overall specificity.

with a specificity of $88.0 \%$ for MRI detectable bladder involvement [7]. Postema et al., found a sensitivity and a specificity of $77.0 \%$ and $97.0 \%$, respectively, for bladder invasion detection by MRI [8]. 5 of eighteen patients, suspected on MRI to have mucosal bladder invasion were not confirmed at histology. Overall MRI specificity and sensibility for the MRI detection of mucosal bladder invasion was 93.5 and $100 \%$ in the 92 patient series [9].

MRI detection of rectal involvement. Rockall et al., found 10 false positives out of 112 patients with $100.0 \%$ sensitivity and $91.0 \%$ specificity [6]. According to Rajaram et al., MRI correctly assessed both spaces with accuracy of $88.9 \%$ in vesicocervical space, and $66.7 \%$ for rectovaginal space [10]. Hricak et al., showed that neither CAT nor MRI were able to detect four cases presenting with tumor in the rectum [11]. Hertel et al., calculated a MRI sensitivity of $50.0 \%$ and a specificity of $86.0 \%$ for rectal invasion [7].

MRI detection of vaginal invasion. Choi et al., [12] in 23 patients reported a sensitivity of $87.0 \%$ and a specificity of $79.0 \%$ with 3 false negatives. Sheu et al., [13] identified vaginal involvement in 9 patients, with 2 false negatives and 6 false positives at histological comparison. The sensitivity was $82.0 \%$ and specificity $84.0 \%$. Errors in the assessment of vaginal invasion occurred in 8 cases out of 11. In the Sheu's study [14], overall sensitivity and specificity for vaginal invasion detection were $75.0 \%$ and $88.0 \%$, respectively.
Uterine body involvement cannot be established by the examining gynaecologist. Sahdev et al., [15] found, for internal os involvement detection, a MRI sensitivity of $90.0 \%$ and a specificity of $98.0 \%$.

MRI detection of myometrial invasion. This was observed through MRI by Sahdev et al., [15] with a sensitivity of $100.0 \%$ and a specificity of $99.0 \%$. MRI correctly detected all of the 7 tumors extending to the endometrium on 150 patients enrolled in his study. According to Wang et al., [16], tumors extending to the uterine body were identified by MRI in 3 out of 4 patients. In one case microscopic extension to the endometrium was not detected by MRI. Yoo et al., [17] studied 99 patients and reported sensitivity and specificity for deep myometrial invasion MRI detection of $46.6 \%$, and $84.5 \%$, respectively.

Primary tumor detection by MRI. Sahdev et al., [15] pointed out a 20/60 incidence of suspect lesions at MRI when no tumor was histologically detectable and suggested scar lesions as the culprit. The false negative rate, for the detection of small tumors $(<1 \mathrm{~cm}$ diameter), was $50.0 \%$ (14/28). Fischerova et al., [18] observed a tumor detection sensitivity of $44 \%$ at MRI, with a specificity of 94.3\% for tumor bulks $\leq 1 \mathrm{~cm}^{3}$. Itoh et al., [19] set tumor detection sensitivity at $75.0 \%$ in a stage I patient. Lesions over $2 \mathrm{~cm}$ were detected in $83.0 \%$ of cases and in $60 \%$ under $2 \mathrm{~cm}$. de Souza et al., [20] observed a tumor detection sensitivity of $87.0 \%$ and specificity of $65.0 \%$ in 43 
patients with tumor volumes of $\leq 1 \mathrm{~cm}^{3}$. Sensitivity and specificity in patients with conization were $95.1 \%$ and $57.9 \%$, respectively, while the same values in patients without conization were $98.2 \%$, and $66.7 \%$, respectively.

According to Sahdev et al., [15], whose study included microscopic stage Ia tumors, detection sensitivity and specificity of the primary tumor was $65.0 \%$ and $77.0 \%$, respectively. The total false-positive MRI rate was $30.6 \%$ $(19 / 62)$ possibly due to tissue post-biopsy oedema or chronic inflammation. Fischerova et al., [18], had a more favourable experience and found MRI sensitivity for tumor detection of $82.9 \%$, with a specificity of $84.2 \%$. de Souza et al., [20] found false positives in 9 over 119 cases with an overall sensitivity of $96.9 \%$ and a specificity of $59.1 \%$. Total results are influenced by low MRI performance in case of small primary tumors.

Tumor size estimation. According to Sahdev et al., [15], there is an average tumor size estimation error of 9 $\mathrm{mm}$, with $95 \%$ limits of agreement from $12.6 \mathrm{~mm}$ to 13.0 $\mathrm{mm}$ when compared with histology. Error drops to $3 \mathrm{~mm}$ if the tumor exceeds $10 \mathrm{~mm}$. Park et al., [21] showed a volume estimation error of $71.9 \%$ if the lesion was confined to the cervix and up to $18.8 \%$ if extending to the vagina. Sheu et al., [13], for tumors over $1 \mathrm{~cm}$, observed the overestimation of tumor size in 3 patients: this was due to inability to differentiate tumor from tissue oedema resulting from previous biopsy or previous cone biopsy. In two cases tumor size was underestimated and errors in measurement were due to indistinct lesion margin. According to Sheu et al., [14], tumor size detected by MRI was $3.23 \pm 1.75 \mathrm{~cm}$ (mean \pm standard deviation) compared with $2.79 \pm 1.76 \mathrm{~cm}$ at pathology evaluation. Over-estimation of tumor size was consistent in tumors larger than $10 \mathrm{~mm}$.

\section{Discussions}

Routine MRI equipment has been updated periodically, but no trend towards a better diagnostic accuracy has been found [22], and, in all the papers we reviewed, the authors used standard 1.5 Tesla equipment. Advanced MRI techniques, like ultra-small particles of iron oxide (USPIO) [23], endorectal/phased-array coils [16], contrast media enhancement [14] and others (endovaginal opacification [15], dynamic MRI imaging, short tau inversion recovery [STIR] sequence [5]) have been used in a limited number of studies but are not addressed by our review. All these techniques might improve diagnostic accuracy of MRI studies. MRI results vary according to the radiologist's experience $[8,22]$.

Only the results with routine MRI in a clinical setting have been considered in this paper.

The overall patient sample set is biased [23] as patients with inoperable tumors were excluded. However, it should be noted that bulky tumors were well represented in all reviewed papers. Tissue preparation for histology could possibly lead to dehydration and shrinkage of the tumor mass. MRI overestimation and underestimation of tumor volume were equally represented in the available data.

Tumor detection and size estimation. MRI evaluation does not precisely correlate with histologic tumor size unless the tumor is bulky (at least $1 \mathrm{~cm}$ diameter) [24]. Tumor size estimation by MRI [25] seems accurate in Ib to IIb stages, with a correlation of $0.93 \%$. The problems observed with MRI evaluation of primary tumor size are: 1) no detection; 2) impossibility of margin definition; 3) confusion with peritumoral reaction or scars. The issue is possibly relevant only if the tumor is confined to the cervix (Figures 1(A)-(B)). The incidence of false positive diagnoses with MRI in Ia1 tumors was 33.0\% (20/60), while false negatives reached $50.0 \%$ [12]. Correct diagnoses were mostly obtained in cases with a previous cone biopsy suggesting scar and not tumor detection. Some recent data reported by Fischerova et al., are encouraging [18] showing an overall sensitivity of $82.9 \%$ and a specificity of $84.2 \%$ in primary tumor detection. For volumes under $1 \mathrm{~cm}^{3}$, the values became $44.0 \%$ and $94.3 \%$, respectively.

Internal os involvement and myometrial invasion. Preliminary data suggest good results with MRI in the definition of cervical internal os involvement. Myometrial and endometrial invasion detection is possible by MRI (Figure 1(D)) [9]. This issue is not unrelated to tumor bulk. Some lesions misdiagnosed as bulky first stages (Figure 1(C)), with unfavourable outcome might include relatively more advanced cases with uterine body invasion demonstrable identified by MRI. Bipat et al., [26] in 21 patients shows that MRI has a good chance of visualizing the internal os. No false-negatives for involvement of the uterine corpus and 3 false positives with suspect uterine body invasion were observed by the authors.

Lymph node involvement. Lymph node invasion is difficult to determine with certainty by MRI and no definite cut-off has been established as regards volume or maximum diameter of nodes.

Criteria for nodal involvement are not standardized. Sahdev et al., [15] suggests that the prediction of nodal status was most accurate when a $9 \mathrm{~mm}$ cut-off was applied to the short-axis diameter of the lymph node [27]. de Souza et al., [20], using a cut-off volume of $5.2 \mathrm{~cm}^{3}$, observed that lymph node metastasis could be predicted with a $78.6 \%$ sensitivity and a $72.5 \%$ specificity. A cutoff of $2.8 \mathrm{~cm}^{3}$ improves sensitivity to $89.0 \%$ but this gives a poor specificity of only $67.0 \%$. Park et al., [21] reports that using $1 \mathrm{~cm}$ diameter cut-off sensitivity and specificity were $57.0 \%$ and $73.0 \%$, respectively, while Narayan et al., [28] with the same cut-off observes a MRI sensitivity of $50.0 \%$. MRI apparently might fail 

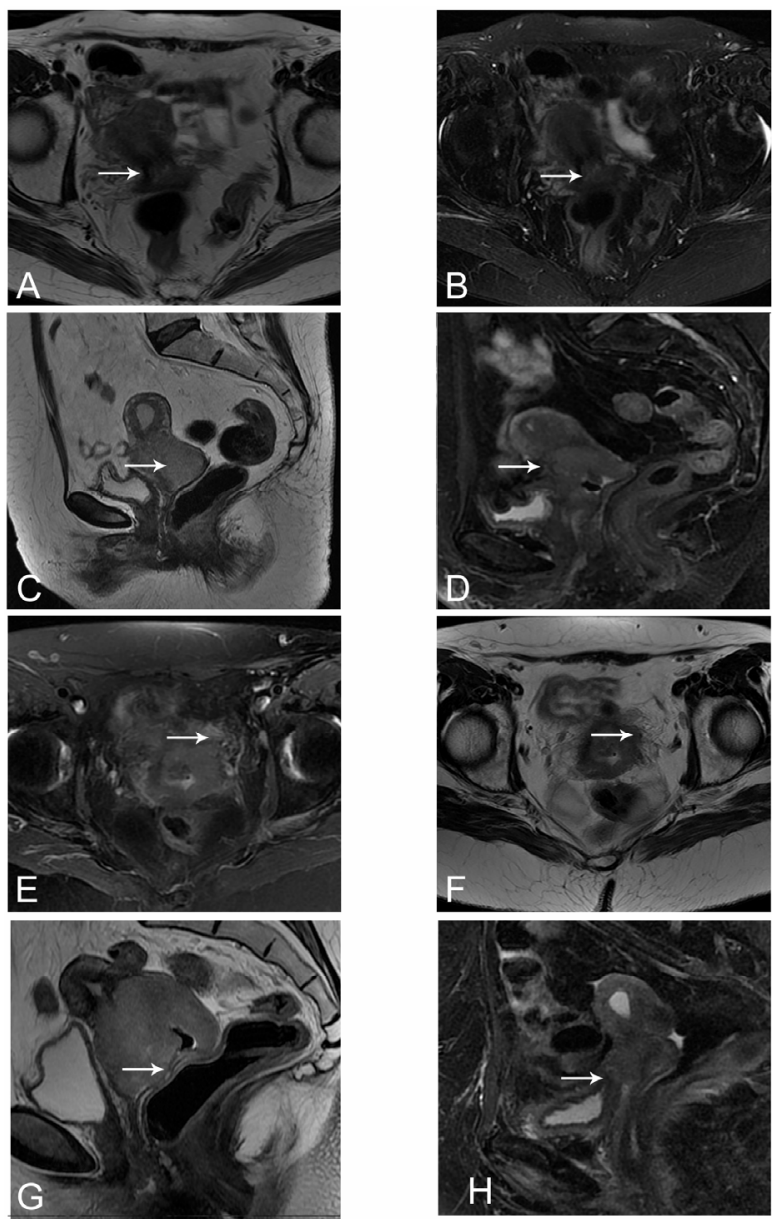

Figure 1. (A and B, respectively) T2 weighted (T2w) pulse sequence MRI showing uterine cervical cancer detection and invasion. A small lesion confined to the cervix, evaluated through axial $\mathrm{T} 2 \mathrm{w}$ and $\mathrm{T} 2 \mathrm{w}$ fat-saturated images. (C) A bulky cervical lesion, invading posterior fornix without rectal or bladder invasion, assessed by sagittal T2w MRI. (D) Cervical cancer with myometrial, (E-F) parametrial, (G) rectal (H) and vaginal invasion detected by: 1) (D) T2w fat-saturated MRI; 2) (E and F, respectively) axial T2w and T2w fat-saturated MRI; 3) (G and $H$ ) sagittal T2w fatsaturated MRI.

because lymph node size can be unaffected in early invasion and large nodes are observed in association with incidental clinical conditions. Sheu et al., [14] found 2 false negatives and 2 false positives, with $71.0 \%$ sensitivity and $92.0 \%$ specificity. Enlargement of lymph nodes due to reactive hyperplasia rather than tumor metastases was noted in 2 patients. In 3 out of 35 women micrometastatic disease was missed by MRI, and 4 patients with abnormally enlarged lymph nodes at MR imaging had negative findings at histological examination. The sensitivity and specificity were $73.0 \%$ and $83.0 \%$, respectively [29]. Hong et al., indicate that MRI lymph node detection could be related to tumor bulk. The sensitivity and specificity were $7 \%$ and $99.3 \%$ in the nonbulky tu- mor group, $43.8 \%$ and $97.1 \%$ in the bulky tumor group [30].

Comparison of proximal and distal node detection. Some preliminary findings suggest better MRI results for lower pelvis metastatic node detection. Choi et al., [12] confirmed the presence of lymph node metastasis in 22 pelvic regions in 17 patients with a sensitivity of $36.0 \%$ and a specificity of $97.0 \%$. Of 3 patients with suspect para-aortic lymph nodes metastases at MRI, surgical resection proved metastases in 2 with a sensitivity of $100.0 \%$ and with 1 false positive due to previous tuberculous lymphadenitis. According to Bellomi et al., [31], MRI sensitivity and specificity in detecting metastatic lymph nodes in the lower pelvis were $80.0 \%$ and $88.0 \%$, respectively, while were $37.5 \%$ and $97.5 \%$ for upper iliac nodes. Overall MRI sensitivity was $72.9 \%$ and specificity 93.1\%. Hertel et al., [7] analyzed para-aortic and pelvic lymph nodes separately, with a MRI sensitivity of $25.0 \%$ for the pelvic region and of $0 \%$ for the para-aortic region. The specificity was $87.0 \%$ and $98.0 \%$. Yoo et al., [17] reported 99 patients and showed sensitivity and specificity for MRI in determining pelvic lymph node metastases of $77.7 \%$ and $85.6 \%$, respectively. MRI failed to detect the presence of paraaortic lymph node metastases in all of the three patients with positive nodes. Discrepancies between MRI and surgical findings could be explained by new lesions appearing in previously apparently unaffected lymph nodes.

Parametrial invasion. MRI sensitivity and specificity in the detection of parametrial invasion indicates problems in the differentiation between abnormal and simply reactive tissue (Figures 1(E)-(F)).

Recent studies suggest an even better specificity value for parametrial involvement detection. Fujiwara et al., [4] demonstrated a sensitivity of $67.8 \%$ and a specificity of $95.7 \%$ for parametrial involvement detection. Fischerova et al., [18], showed a sensitivity of $50.0 \%$, and a specificity of $97.8 \%$, respectively, for parametrial invasion. If the cervical stromal ring appears intact at MRI, parametrial extension has been reported in 0 to $6 \%$ of cases [25].

Parametrial involvement and tumor bulk. Hricak et al., [11] focused on MRI capability to detect advanced cancer stage ( $\geq \mathrm{IIb})$ showing a sensitivity of $53.0 \%$ and a specificity of $75.0 \%$. Jena et al., [32] observed that tumor volume at MRI in parametrial invasion cases ranges from $17.3 \mathrm{~cm}^{3}$ to $99.2 \mathrm{~cm}^{3}$. The best sensitivity of $59.3 \%$ and specificity of $61.5 \%$ was found for a tumor volume of $36.4 \mathrm{~cm}^{3}$. Park et al., [21] reported an accuracy of $84.4 \%$ compared with surgical specimen findings, with only 2 patients up-staged $(6.3 \%)$ but 3 down-staged $(9.4 \%)$ including one with no visible tumor at MRI. Choi et al., [12] showed a sensitivity of $38.0 \%$ for 226 parametria analyzed, with an accuracy of $94.0 \%$. It may be that 
specificity is negatively affected by increasing tumor bulk. According to Testa et al., MRI provided low sensitivity $(2 / 5,40.0 \%)$ for the presence of parametrial infiltration [33].

MRI interpretation might very according to different radiologists. Reviewing parametrial invasion, Postema et al. [8], showed that reader nr. 1 reported a sensitivity of $20.0 \%$ and a specificity of $97.0 \%$, while reader nr. 2 observed a sensitivity and a specificity of $60.0 \%$ and $73.0 \%$, respectively.

Bladder, rectal and vaginal invasion. Data on rectal invasion detected by MRI in cervical cancer patients are scant and conflicting. Sensitivity varies from 50 to $100 \%$ in limited reports. MRI overdiagnoses bladder and rectal invasion. MRI might lead to detection of clinically unsuspected lesions and has determined restaging in one instance [14]. Absolute sensitivity and specificity for MRI detection of rectal invasion (Figure 1(G)) seems to be aligned to those observed in parametrial tumor detection. Vaginal invasion might possibly be underdiagnosed by MRI (Figure 1(H)).

\section{Conclusions}

MRI use is encouraged for cervical cancer staging. There seems to be good correlation between histological and MRI tumor bulk. There is a high probability of non extension to parametria if cervical integrity is observed at MRI and good indication of extension to proximal structures. Primary preoperative evaluation of uterine cancer by MRI does not accurately predict the nodal status and the degree of myometrial and parametrial invasion [17], but MRI shows encouraging progress in the detection of uterine myometrial and endometrial invasion, where CAT scans mostly fail.

MRI has been proposed as a substitute for invasive cystoscopy and proctoscopy in the initial screening of cervical cancer and preliminary data seem encouraging.

In general, the role of modern imaging techniques still remains undefined in the current management of uterine cervical cancer. MRI and CAT have been approved for use of initial staging in patients with cervical cancer by Medicare and Medicaid Services [33]. Due to its good tissue contrast, MRI is the preferred imaging technique for tumor detection and invasion evaluation in advanced stage disease [22]. In patients with negative results by MRI and CAT, the use of PET has been approved by health care providers such as Medicare [34]. A preliminary report by Testa et al., [33] shows that Ultrasound (US) and MRI has similar sensitivity and specificity in preoperative staging. US has the advantages over MRI of low cost and widespread availability.

Despite all limitations, introduction of MRI has improved staging in cervical cancer patients according to Chung et al., [3] and the American College of Radiology
Imaging Network (ACRIN) study [11], from 30\% - 40\% to about $70 \%$ accuracy [17].

Selman et al., [35] reports a pooled negative likelihood ratio of $50 \%$ for lymph-node metastasis detection by MRI in a meta-analysis of the literature. Adding a sentinel lymph node biopsy can raise the accuracy to $94 \%$. Intraoperative histology could lead to a better primary staging of uterine cervical cancer. According to Rajaram, the incidence of positive nodes in stage IB patients, is approximately $15 \%$ [10]. Thus sentinel lymph node (SLN) evaluation, is a practical approach to reduce extensive lymph node dissection.

SLN evaluation is an established technique for melanoma and breast carcinoma staging and its use in vulva, lung and cervical carcinoma treatment is being actively discussed [36-39]. A combined imaging and histology approach might be warranted in the management of uterine cervical carcinoma.

Awareness of MRI limitations is crucial in primary cervical cancer staging.

\section{Acknowledgements}

Grant of "Fondazione Enrico ed Enrica Sovena" to C.V.

\section{REFERENCES}

[1] A. Jemal, R. Siegel, E. Ward, T. Murray, J. Xu and M. J. Thun, "Cancer Statistics," CA: A Cancer Journal of Clinicians, Vol. 57, No. 1, 2007, pp. 43-66. doi:10.3322/canjclin.57.1.43

[2] H. J. Choi, W. Ju, S. K. Myung and Y. Kim, "Diagnostic Performance of Computed Tomography, Magnetic Resonance Imaging, and Positron Emission Tomography or Positron Emission Tomography/Computer Tomography for Detection of Metastatic Lymph Nodes in Patients with Cervical Cancer: Meta-Analysis," Cancer Science, Vol. 10, No. 6, 2010, pp. 1471-1479. doi:10.1111/j.1349-7006.2010.01532.x

[3] H. H. Chung, S. B. Kang, J. Y. Cho, J. W. Kim, N. H. Park, Y. S. Song, S. H. Kim and H. P. Lee, "Can Preoperative MRI Accurately Evaluate Nodal and Parametrial Invasion in Early Stage Cervical Cancer?" Japanese Journal of Clinical Oncology, Vol. 37, No. 3, 2007, 370375. doi:10.1093/jico/hym036

[4] K. Fujiwara, E. Yoden, T. Asakawa, M. Shimizu, M. Hirokawa, Y. Mikami, T. Oda, I. Joja, Y. Imajo and I. Kohno, "Negative MRI Findings with Invasive Cervical Biopsy May Indicate Stage IA Cervical Carcinoma," Gynecoloy Oncology, Vol. 79, No. 3, 2000, pp. 451-456. doi:10.1006/gyno.2000.5967

[5] W. W. M. Lam, N. M. C. So, W. T. Yang and C. Metreweli, "Detection of Parametrial Invasion in Cervical Carcinoma: Role of Short Tau Inversion Recovery Sequence," Clinical Radiology, Vol. 55, No, 9, 2000, pp. 702-707. doi:10.1053/crad.2000.0506

[6] A. G. Rockall, S. Ghosh, F. Alexander-Sefre, S. Babar, M. T. Younis, S. Naz, I. J. Jacobs and R. H. Reznek, "Can 
MRI Rule Out Bladder and Rectal Invasion in Cervical Cancer to Help Select Patients for Limited EUA?" Gynecology Oncology, Vol. 101, No. 2, 2006, pp. 244-249. doi:10.1016/i.ygyno.2005.10.012

[7] H. Hertel, C. Köhler, T. Elhawary, W. Michels, M. Possover and A. Schneider, "Laparoscopic Staging Compared with Imaging Techniques in the Staging of Advanced Cervical Cancer," Gynecology Oncology, Vol. 87, No. 1, 2002, pp. 46-51. doi:10.1006/gyno.2002.6722

[8] S. Postema, P. T. M. Pattynama, A. Van den Berg-Huysmans, P. W. Lex, G. Kenter and J. B. Trimbos, "Effect of MRI on Therapeutic Decisions in Invasive Cervical Carcinoma. Direct Comparison with the Pelvic Examination as a Preoperative Test," Gynecology Oncology, Vol. 79, No. 3, 2000, pp. 485-489. doi:10.1006/gyno.2000.5986

[9] H. Nam, S. J. Huh, W. Park, D. S. Bae, B. G. Kim, J. H. Lee, C. K. Kim and B. K. Park, "Prognostic Significance of MRI-Detected Bladder Muscle and/or Serosal Invasion in Patients with Cervical Cancer Treated with Radiotherapy," British Journal of Radiology, Vol. 83, No. 994, 2010, pp. 868-873. doi: $10.1259 /$ bjr/6646798

[10] S. Rajaram, H. Sharma, S. K. Bhargava, R. P. Tripathi, N. Goel and S. Mehta, "Mapping the Extent of Disease by Multislice Computed Tomography, Magnetic Resonance Imaging and Sentinel Node Evaluation in Stage I and II Cervical Carcinoma," Journal of Cancer Research and Therapeutics, Vol. 6, No. 3, 2010, pp. 267-271. doi:10.4103/0973-1482.73342

[11] H. Hricak, C. Gatsonis, D. S. Chi, M. A. Amendola, K. Brandt, L. H. Schwartz, S. Koelliker, E. S. Siegelman, J. J. Brown, R. B. McGhee Jr., R. Iyer, K. M. Vitellas, B. Snyder, H. J. Long III, J. V. Fiorica and D. G. Mitchell, "Role of Imaging in Pretreatment Evaluation of Early Invasive Cervical Cancer: Results of the Intergroup Study American College of Radiology Imaging Network 6651Gynecology Oncology Group 183," Journal of Clinical Oncology, Vol. 23, No. 36, 2005, pp. 9329-9337. doi:10.1200/JCO.2005.02.0354

[12] S. H. Choi, S. H. Kim, H. J. Choi, B. K. Park and H. J. Lee, "Preoperative Magnetic Resonance Imaging Staging of Uterine Cervical Carcinoma," Journal of Computer Assisted Tomography, Vol. 28, No. 5, 2004, pp. 620-627.

[13] M. H. Sheu, C. Y, Chang, J. H. Wang and M. S. Yen, "Preoperative Staging of Cervical Carcinoma with MR Imaging: A Reappraisal of Diagnostic Accuracy and Pitfalls," European Radiology, Vol. 11, No. 9, 2001, pp. 1828-1833. doi:10.1007/s003300000774

[14] M. H. Sheu, C. Y. Chang, J. H. Wang and M. S. Yen, "MR Staging of Clinical Stage I and IIa Cervical Carcinoma: A Reappraisal of Efficacy and Pitfalls," European Journal of Radiology, Vol. 38, No. 3, 2001, pp. 225-231. doi:10.1016/S0720-048X(00)00278-3

[15] A. Sahdev, S. A. Sohaib, A. E. T. Wenaden, J. H. Shepherd and R. H. Reznek, "The Performance of Magnetic Resonance Imaging in Early Cervical Carcinoma: A Long Term Experience," International Journal of Gynecological Cancer, Vol. 17, No. 3, 2007, pp. 629-636. doi:10.1111/j.1525-1438.2007.00829.x
[16] L. J. Wang, Y. C. Wong, C. J. Chen, K. G. Huang and S. Hsueh, "Cervical Carcinoma: MR Imaging with Integrated Endorectal/Phased-Array Coils: A Pilot Study," European Radiology, Vol. 11, No. 9, 2001, pp.1822-1827. doi:10.1007/s003300000794

[17] S. C. Yoo, W. Y. Kim, J. H. Yoon, H. Y. Kim, E. J. Lee, S. J. Chang, K. H. Chang and H. S. Ryu, "Accuracy of Preoperative Magnetic Resonance Imaging in Assessing Lymph Node Metastasis and Myometrial Invasion in Patients with Uterine Cancer," European Journal of Gynaecological Oncology, Vol. 30, No. 2, 2009, pp. 167170.

[18] D. Fischerova, D. Cibula, H. Stenhova, H. Vondrichova, P. Calda, M. Zikan, P. Freitag, J. Slama, P. Dundr and J. Belacek, "Transrectal Ultrasound and Magnetic Resonance Imaging in Staging of Early Cervical Cancer," International Journal of Gynecological Cancer, Vol. 18, No. 4, 2008, pp. 766-772. doi:10.1111/j.1525-1438.2007.01072.x

[19] K. Itoh, T. Shiozawa, S. Ohira, S. Shiohara and I. Konishi, "Correlation between MRI and Histopathologic Findings in Stage I Cervical Carcinomas: Influence of Stromal Desmoplastic Reaction," International Journal of Gynecological Cancer, Vol. 16, No. 2, 2006, pp. 610-614. doi:10.1111/j.1525-1438.2006.00383.x

[20] N. M. de Souza, R. Dina, G. A. McIndoe and W. P. Soutter, "Cervical Cancer: Value of an Endovaginal Coil Magnetic Resonance Imaging Technique in Detecting Small Volume Disease and Assessing Parametrial Extension," Gynecology Oncology, Vol. 102, No. 1, 2006, pp. 80-85. doi:10.1016/j.ygyno.2005.11.038

[21] W. Park, Y. J. Park, S. J. Huh, B. G. Kim, D. S. Bae, J. Lee, B. H. Kim, J. Y. Choi, Y. C. Ahn and D. H. Lim, "The Usefulness of MRI and PET Imaging for the Detection of Parametrial Involvement and Lymph Node Metastasis in Patients with Cervical Cancer," Japanese Journal of Clinical Oncology, Vol. 35, No. 5, 2005, pp. 260-264. doi:10.1093/jico/hyi079

[22] K. Hancke, V. Heilmann, P. Straka, R. Kreienberg and C. Kurzeder, "Pre-Treatment Staging of Cervical Cancer: Is Imaging Better Than Palpation? Role of CT and MRI in Preoperative Staging of Cervical Cancer: Single Institution Results of 255 Patients," Annals of Surgical Oncology, Vol. 15, No. 10, 2008, pp. 2856-2861. doi:10.1245/s10434-008-0088-7

[23] A. G. Rockall, S. A. Sohaib, M. G. Harisinghani, S. A. Babar, N. Singh, A. R. Jeyarajah, D. H. Oram, I. J. Jacobs, J. H. Shepherd and R. H. Reznek, "Diagnostic Performance of Nanoparticle-Enhanced Magnetic Resonance Imaging in the Diagnosis of Lymph Node Metastases in Patients with Endometrial and Cervical Cancer," Journal of Clinical Oncology, Vol. 23, No. 12, 2005, pp. 2813-2821. doi:10.1200/JCO.2005.07.166

[24] R. Manfredi, B. Gui, A. Giovanzana, S. Marini, M. Di Stefano, G. Zannoni, G. Scambia and L. Bonomo, "Localized Cervical Cancer (Stage < IIB): Accuracy of MR Imaging in Planning Less Extensive Surgery," $L a R a$ diologia Medica, Vol. 114, No. 6, 2009, pp. 960-975. doi:10.1007/s11547-009-0397-3

[25] E. Sala, S. Wakely, E. Senior and D. Lomas, "MRI of 
Malignant Neoplasms of the Uterine Corpus and Cervix," American Journal of Roentgenology, Vol. 188, No. 6, 2007, pp. 1577-1587. doi:10.2214/AJR.06.1196

[26] S. Bipat, R. A. van den Berg, J. van der Velden, J. Stoker and A. M. Spijkerboer, "The Role of Magnetic Resonance Imaging in Determining the Proximal Extension of Early Stage Cervical Cancer to the Internal OS," European Journal of Radiology, Vol. 78, No. 1, 2009, pp. 60-64. doi:10.1016/j.ejrad.2009.06.006

[27] H. J. Choi, S. H. Kim, S. S. Seo, S. Kang, S. Lee, J. Y. Kim, Y. H. Kim, J. S. Lee, H. H. Chung, J. H. Lee and S. Y. Park, "MRI for Pretreatment Lymph Node Staging in Uterine Cervical Cancer," American Journal of Roentgenology, Vol. 187, No. 5, 2006, pp. 538-543. doi:10.2214/AJR.05.0263

[28] K. Narayan, R. J. Hicks, T. Jobling, D. Bernshaw and A. F. Mckenzie, "A Comparison of MRI and PET Scanning in Surgically Staged Loco-Regionally Advanced Cervical Cancer: Potential Impact on Treatment," International Journal of Gynecological Cancer, Vol. 11, No. 4, 2001, pp. 263-271. doi:10.1046/j.1525-1438.2001.011004263.x

[29] M. J. Reinhardt, C. Ehritt-Braun, D. Vogelgesang, C. Ihling, S. Högerle, M. Mix, E. Moser and T. M. Krause, "Metastatic Lymph Nodes in Patients with Cervical Cancer: Detection with MR Imaging and FDG PET," Radiology, Vol. 218, No. 3, 2001, pp. 776-782.

[30] K. S. Hong, W. Ju, H. J. Choi, J. K. Kim, M. H. Kim and K. S. Cho, "Differential Diagnostic Performance of Magnetic Resonance Imaging in the Detection of Lymph Node Metastases According to the Tumor Size in EarlyStage Cervical Cancer Patients," International Journal of Gynecological Cancer, Vol. 20, No. 5, 2010, pp. 841-846. doi:10.1111/IGC.0b013e3181db5140

[31] M. Bellomi, G. Bonomo, F. Landoni, G. Villa, M. E. Leon, L. Bocciolone, A. Maggioni and G. Viale, "Accuracy of Computed Tomography and Magnetic Resonance Imaging in the Detection of Lymph Node Involvement in Cervix Carcinoma," European Radiology, Vol. 15, No. 12, 2005, pp. 2469-2474. doi:10.1007/s00330-005-2847-1

[32] A. Jena, R. Oberoi, S. Rawal, S. K. Das and K. K. Pandey, "Parametrial Invasion in Carcinoma of Cervix: Role of MRI Measured Tumour Volume," British Journal of Radiology, Vol. 78, No. 936, 2005, pp. 1075-1077. doi: $10.1259 / \mathrm{bjr} / 36116150$

[33] A. C. Testa, M. Ludovisi, R. Manfredi, G. Zannoni, B. Gui, D. Basso, A. Di Legge, A. Licameli, R. Di Bidino, G. Scambia and G. Ferrandina, "Transvaginal Ultrasonography and Magnetic Resonance Imaging for Assessment of preseNce, Size and Extent of Invasive Cervical Cancer," Ultrasound in Obstetrics and Gynecology, Vol. 34, No. 3, 2009, pp. 335-344. doi:10.1002/uog.7325

[34] Conference Report, "The Contribution of New Imaging
Techniques in Staging Cervical Cancer," Gynecology Oncology, Vol. 107, Suppl. 1, 2007, pp. S10-S12.

[35] T. J. Selman, C. Mann, J. Zamora, T. L. Appleyard and K. Khan, "Diagnostic Accuracy of Test for Lymph-Node Status in Primary Cervical Cancer: A Systematic Review and Meta-Analysis," Canadian Medical Association Journal, Vol. 25, No. 7, 2008, pp. 855-862. doi:10.1503/cmaj.071124

[36] D. L. Morton, J. F. Thompson, R. Essner, R. Elashoff, S. L. Stern, O. E. Nieweg, D. F. Roses, C. P. Karakousis, N. Mozzillo, D. Reintgen, H. J. Wang, E. C. Glass and A. J. Cochran, "Validation of the Accuracy of Intraoperative Lymphatic Mapping and Sentinel Lymphadenectomy for Early-Stage Melanoma: A Multicenter Trial. Multicenter Selective Lymphadenectomy Trial Group," Annals of Surgery, Vol. 230, No. 4, 1999, pp. 453-463. doi:10.1097/00000658-199910000-00001

[37] K. M. McMasters, S. L. Wong, C. Chao, C. Woo, T. M. Tuttle, R. D. Noyes, D. J. Carlson, A. L. Laidley, T. Q. McGlothin, P. B. Ley, C. M. Brown, R. L. Glaser, R. E. Pennington, P. S. Turk, D. Simpson, M. J. Edwards and University of Louisville Breast Cancer Study Group, "Defining the Optimal Surgeon Experience for Breast Cancer Sentinel Lymph Node Biopsy: A Model for Implementation of New Surgical Techniques," Annals of Surgery, Vol. 234, No. 3, 2001, pp. 292-299. doi:10.1097/00000658-200109000-00003

[38] C. Levenback, T. W. Burke, D. M. Gershenson, M. Morris, A. Malpica and M. I. Ross, "Intraoperative Lymphatic Mapping for Vulvar Cancer," Obstetrics and Gynecology, Vol. 84, No. 2, 1994, pp. 163-167.

[39] M. J. Liptay, G. A. Masters, D. J. Winchester, B. L. Edelman, B. J. Garrido, T. R. Hirschtritt, R. M. Perlman and W. A. Fry, "Intraoperative Radioisotope Sentinel Lymph Node Mapping in Non-Small Cell Lung Cancer,' The Annals of Thoracic Surgery, Vol. 70, No. 2, 2000, pp. 384-389. doi:10.1016/S0003-4975(00)01643-X

[40] W. P. Soutter, J. Hanoch, T. D’Arcy, R. Dina, G. A. McIndoe and N. M. DeSouza, "Pretreatment Tumour Volume Measurement on High-Resolution Magnetic Resonance Imaging as a Predictor of Survival in Cervical Cancer," British Journal of Obstetrics and Gynaecology, Vol. 111, No. 7, 2004, pp. 741-747. doi:10.1111/j.1471-0528.2004.00172.x

[41] H. J. Choi, J. W. Roh, S. S. Seo, S. Lee, J. Y. Kim, S. K. Kim, K. W. Kang, J. S. Lee, J. Y. Jeong and S. Y. Park, "Comparison of the Accuracy of Magnetic Resonance Imaging and Positron Emission Tomography/Computed Tomography in the Presurgical Detection of Lymph Node Metastases in Patients with Uterine Cervical Carcinoma," Cancer, Vol. 106, 4, 2006, pp. 914-922. doi:10.1002/cncr.21641 